Applied and

NISTIR 89-4102

Computational

Mathematics

Division

Center for Computing and Applied Mathematics

\title{
The Computation and Use of the Asymptotic Covariance Matrix for Measurement Error Models
}

Paul T. Boggs and Janet E. Rogers

\author{
June, 1989 \\ (Revised October, 1990)
}

\section{U.S. DEPARTMENT OF COMMERCE}

National Institute of Standards and Technology

Gaithersburg, MD 20899 


\title{
The Computation and Use of the Asymptotic Covariance Matrix for Measurement Error Models*
}

\author{
Paul T. Boggs ${ }^{\dagger} \quad$ Janet E. Rogers ${ }^{\ddagger}$
}

\begin{abstract}
Measurement error models assume that errors occur in both the response and predictor variables. In using these models, confidence regions and intervals for the model parameters are frequently of interest. We discuss the solution of the minimization problem resulting from the use of a particular class of measurement error models, and we develop a procedure for accurately computing an asymptotic form for the covariance matrix that can be used to construct approximate confidence regions and intervals. We then show via a Monte Carlo study that the quality of the confidence regions and intervals constructed from this matrix can be quite good.

Keywords: confidence intervals, confidence regions, covariance matrix, errors in variables, measurement error models, Monte Carlo study, ordinary least squares, orthogonal distance regression, simultaneous equations models.
\end{abstract}

\section{Introduction}

Parameter estimation and data fitting are among the most common activities in science, with the ordinary least squares criterion being by far the most frequently

${ }^{*}$ Contribution of the National Institute of Standards and Technology and not subject to copyright in the United States.

$\dagger$ Applied and Computational Mathematics Division, National Institute of Standards and Technology, Gaithersburg, MD 20899. INTERNET: boggs@cam.nist.gov

${ }^{\ddagger}$ Applied and Computational Mathematics Division, National Institute of Standards and Technology, Boulder, CO, 80303-3328. INTERNET: jrogers@cam.nist.gov 
used. The emergence over the last decade of high quality software for finding the ordinary least squares solution for nonlinear functions has allowed researchers to consider more realistic data fitting and parameter estimation models for many situations. Until recently, however, researchers have not had procedures available that would take into account the fact that the errors in the observations of the predictor variable, $x_{i}$, are frequently significant with respect to the errors in the observations of the response variable, $y_{i}$. Such errors can make a substantial difference in resulting estimations. (See, e.g., [Boggs et al., 1988], and [Fuller, 1987].)

To be specific, ordinary least squares problems arise when the actual (or true) value of the response variable, denoted by a superscript $a$, i.e., $y_{i}^{a}$, is observed with some actual but unknowable error $\epsilon_{i}^{a}$, while the predictor variable is observed without error, i.e., $x_{i}=x_{i}^{a}$. Now if we assume that

$$
\begin{aligned}
y_{i} & =y_{i}^{a}-\epsilon_{i}^{a} \\
& =f\left(x_{i}^{a} ; \beta^{a}\right)-\epsilon_{i}^{a} \quad i=1, \ldots, n,
\end{aligned}
$$

where $\beta^{a}$ denotes the actual value of the vector of model parameters, then the ordinary least squares criterion, which minimizes the sum of the squares of the estimates of the errors in $y_{i}$, can be applied to obtain an estimate of $\beta^{a}$.

If there is also a significant actual, but unknowable, error $\delta_{i}^{a}$ in the predictor variable, so that $x_{i}=x_{i}^{a}-\delta_{i}^{a}$, then a generalization of the ordinary least squares criterion is required, since, in this case the model becomes

$$
y_{i}=f\left(x_{i}+\delta_{i}^{a} ; \beta^{a}\right)-\epsilon_{i}^{a} \quad i=1, \ldots, n .
$$

This problem goes under various names, including errors in variables, generalized least squares, orthogonal distance regression, and measurement error models. We prefer measurement error models in deference to the book of Fuller [1987] that presents the definitive modern treatment of the problem. We also use the term orthogonal distance regression since, as we show in $\S 2$, it is a useful geometric description of the problem actually solved.

As in the ordinary least squares case, when using measurement error models one is frequently interested in constructing confidence regions and/or confidence intervals for the model parameters. To this end, Fuller [1987] derives the asymptotic form of the covariance matrix and uses it in several examples. It is well known, however, that for nonlinear models in general, and for measurement error models in particular, confidence regions and intervals constructed using the covariance matrix are only approximate. 
In this paper we discuss a stable and efficient numerical computation of the covariance matrix of the estimators of the parameters of measurement error models, and the use of this matrix to construct confidence regions and/or confidence intervals. The quality of confidence regions and intervals for nonlinear ordinary least squares estimators was discussed in an extensive study by Donaldson and Schnabel [1987]. Here, we report on the quality of the confidence regions and intervals obtained for only four measurement error models. Our study demonstrates that these approximate regions and intervals can be quite good.

In $\S 2$ we give the details of the measurement error model and the formulation of the minimization problem to be solved. We briefly review the solution of this problem using the numerically stable and efficient algorithm that is provided by Boggs et al. [1987] and implemented in ODRPACK [Boggs et al., 1989].

In $\S 3$ we review the basis for using the covariance matrix for estimating confidence regions and intervals. In $\S 4$ we show how the covariance matrix can be efficiently computed in a numerically stable manner, as has been done in ODRPACK. Finally, in $§ 5$, we present the description and results of our Monte Carlo study of the accuracy of the confidence regions and intervals obtained using the covariance matrix.

Despite its potential inaccuracy, the covariance matrix is frequently used to construct confidence regions and intervals for both nonlinear ordinary least squares and measurement error models because the resulting regions and intervals are inexpensive to compute, often adequate, and familiar to practitioners. Caution must be exercised when using such regions and intervals, however, since the validity of the approximation will depend on the nonlinearity of the model, the variance and distribution of the errors, and the data itself. When more reliable intervals and regions are required, other more accurate methods should be used. (See, e.g., [Donaldson and Schnabel, 1987], and [Efron, 1985].)

\section{Measurement Error Models and Orthogonal Distance Regression}

In this section we show that the measurement error problem can be viewed as a generalization of the nonlinear ordinary least squares problem. We then briefly discuss its efficient solution.

The data fitting problem that we consider is composed of an observed data set $\left(x_{i}, y_{i}\right), i=1, \ldots, n$, and a model that is purported to explain the relationship of the response variable $y_{i} \in \Re^{1}$ to the predictor variable $x_{i} \in \Re^{m}$. We assume that 
the response variable is given as a function of the predictor variable and a set of parameters $\beta \in \Re^{p}$, i.e.,

$$
y_{i}^{a}=f\left(x_{i}^{a} ; \beta^{a}\right) \quad i=1, \ldots, n,
$$

where $f$ is a smooth function that can be either linear or nonlinear in $x_{i}$ and $\beta$, and where the superscript $a$ denotes the actual (or true) value of the corresponding quantity.

The explicit measurement error model results when we allow additive errors in both $x_{i}$ and $y_{i}$. If we assume that $y_{i}=y_{i}^{a}-\epsilon_{i}^{a}$ and $x_{i}=x_{i}^{a}-\delta_{i}^{a}$, where $\delta_{i}^{a} \in \Re^{m}$ is the actual, but unknown, additive error in the observation $x_{i}$, then the observations satisfy

$$
y_{i}=f\left(x_{i}+\delta_{i}^{a} ; \beta^{a}\right)-\epsilon_{i}^{a} \quad i=1, \ldots, n .
$$

The term explicit refers to the fact that $y$ can be written directly as a function of $x$ and $\beta$. The more general implicit problem, which has the form

$$
\bar{f}\left(x_{i}+\delta_{i}^{a}, y_{i}+\epsilon_{i}^{a} ; \beta^{a}\right)=0,
$$

is considered in [Fuller, 1987]. The implicit problem is computationally more difficult, and is not discussed further here. (See, e.g., [Boggs et al., 1987].)

When there are errors in both $x_{i}$ and $y_{i}$, then it is reasonable to define the distance from the observation $\left(x_{i}, y_{i}\right)$ to the curve $f(x ; \beta)$ as the radius of the smallest circle centered at $\left(x_{i}, y_{i}\right)$ that is tangent to the curve. If the point of tangency is $\left(x_{i}+\delta_{i}, y_{i}+\epsilon_{i}\right)$, then, by the Pythagorean theorem, the orthogonal distance is

$$
r_{i}^{2}=\left(f\left(x_{i}+\delta_{i} ; \beta\right)-y_{i}\right)^{2}+\delta_{i}^{\mathrm{T}} \delta_{i},
$$

where superscript $\mathrm{T}$ denotes transpose.

The observations $x_{i}$ and $y_{i}$ can have unequal precision, however. We compensate for this by generalizing (2.1) to the weighted orthogonal distance,

$$
\tilde{r}_{i}^{2}=\left(f\left(x_{i}+\delta_{i} ; \beta\right)-y_{i}\right)^{2}+\delta_{i}^{\mathrm{T}} d_{i}^{2} \delta_{i},
$$

where $d_{i} \in \Re^{m \times m}, i=1, \ldots, n$, is a set of positive diagonal matrices that weight each individual component of $\delta_{i}$.

We can then approximate $\beta^{a}$ by finding that $\beta$ which minimizes the sum of the squares of the $\tilde{r}_{i}$. That is, we solve

$$
\min _{\beta, \delta} \sum_{i=1}^{n} w_{i}^{2}\left[\left(f\left(x_{i}+\delta_{i} ; \beta\right)-y_{i}\right)^{2}+\delta_{i}^{\mathrm{T}} d_{i}^{2} \delta_{i}\right]
$$


where $w_{i}, i=1, \ldots, n$, is again a set of nonnegative numbers that allows us to vary the contribution of the various observations to the sum of squares.

The orthogonal distance regression problem defined by (2.2) can also be expressed as a nonlinear ordinary least squares problem with $n+n m$ observations and $p+n m$ unknowns. We designate the unknowns of this ordinary least squares problem as $\eta^{\mathrm{T}}=\left(\beta^{\mathrm{T}}, \delta_{1}^{\mathrm{T}}, \ldots, \delta_{n}^{\mathrm{T}}\right)$, and the sum of squares to be minimized is

$$
S(\eta) \equiv G(\eta)^{\mathrm{T}} \Omega G(\eta)
$$

where $G(\eta)$ is the vector valued function whose $i$ th element is defined by

$$
g_{i}(\eta)= \begin{cases}f\left(x_{i}+\delta_{i} ; \beta\right)-y_{i} & i=1, \ldots, n, \\ \eta_{p+i-n} & i=n+1, \ldots, n+n m,\end{cases}
$$

and $\Omega \in \Re^{(n+n m) \times(n+n m)}$ is the diagonal weighting matrix given by

$$
\Omega=\left[\begin{array}{ll}
W & \\
& D
\end{array}\right]
$$

with $W \in \Re^{n \times n}$ the diagonal matrix with $i$ th component $w_{i}^{2}$, and $D \in \Re^{n m \times n m}$ the diagonal matrix composed of the individual diagonal matrices $w_{i}^{2} d_{i}^{2}$. The ordinary least squares representation of (2.2) is thus

$$
\min _{\eta} S(\eta)=\min _{\eta} \sum_{i=1}^{n+n m} \Omega_{i i} g_{i}(\eta)^{2}
$$

where $\Omega_{i i}$ denotes the $(i, i)$ th element of $\Omega$.

Boggs et al. [1987] have exploited the special structure of the first derivative of $G(\eta)$ with respect to $\eta$ to create a trust-region, Levenberg-Marquardt algorithm for solving the orthogonal distance regression problem defined by (2.2). Their algorithm is both stable and efficient, requiring only $O\left(n p^{2}\right)$ operations per iteration. A similar ordinary least squares algorithm applied to (2.4) would require $O\left(n(n m+p)^{2}\right)$ operations per iteration. Thus the time per iteration in the [Boggs et al., 1987] algorithm grows linearly in $n$ while it grows as $n^{3}$ in an ordinary least squares code applied to (2.4). The portable Fortran subroutine library ODRPACK [Boggs et al., 1989] is an implementation of this algorithm. ODRPACK can thus be used to solve much larger orthogonal distance regression problems than could be solved using nonlinear ordinary least squares software, even though both solutions are mathematically equivalent. ODRPACK is available free of charge from the authors. 


\section{Linearized Confidence Regions and Intervals}

Confidence regions and confidence intervals are commonly computed in statistical applications to assess a bound on the expected difference between the estimated value and the actual (or true) value. Methods of constructing $100(1-\alpha) \%, 0<$ $\alpha<1$, confidence regions and intervals that are statistically guaranteed to contain the true value $100(1-\alpha) \%$ of the time are called exact; all other methods are called approximate. When $f(x ; \beta)$ is nonlinear, construction of exact confidence regions and confidence intervals is difficult, and so approximate methods are frequently used (see, e.g., [Donaldson and Schnabel, 1987], or [Draper and Smith, 1981]).

For nonlinear functions, the most easily computed and most widely used of these approximate methods is the linearization method, which assumes that the nonlinear function can be adequately approximated at the solution by a linear model. The adequacy of these approximations will depend on how well the linearized model approximates the actual function over the region defined by the linearized confidence region and confidence intervals. This, in turn, depends on the nonlinearity of the function [Bates and Watts, 1980], and the residual variance. Donaldson and Schnabel [1987] have shown that linearized confidence intervals are often good in practice, while linearized confidence regions tend to be inadequate.

The linearized confidence regions and intervals for the $\beta \mathrm{s}$ and the $\delta$ s estimated by orthogonal distance regression are the same as the linearized regions and intervals that would be obtained if the orthogonal distance regression problem were solved as a $p+n m$ parameter nonlinear ordinary least squares problem (see $\S 2$ ). That is, one assumes a linear approximation to the nonlinear function at the solution is adequate, and that $\left(\epsilon^{\mathrm{T}}, \delta_{1}^{\mathrm{T}}, \ldots, \delta_{n}^{\mathrm{T}}\right)^{\mathrm{T}} \sim \mathrm{N}\left(0,\left(\sigma^{a}\right)^{2} \Omega^{-1}\right)$, where $\left(\sigma^{a}\right)^{2}$ is the true residual variance, estimated by $\hat{\sigma}^{2}=G(\hat{\eta})^{\mathrm{T}} \Omega G(\hat{\eta}) /(n-p)$. Then a $100(1-\alpha) \%$ linearized confidence region for $\eta^{a}$ can be specified as the region that contains those values $\eta$ for which

$$
(\eta-\hat{\eta})^{\mathrm{T}} V^{-1}(\eta-\hat{\eta}) \leq p \mathrm{~F}_{p, n-p, 1-\alpha},
$$

and a $100(1-\alpha) \%$ linearized confidence interval for $\eta_{j}^{a}$ can be specified as the interval

$$
\left|\eta_{j}-\hat{\eta}_{j}\right| \leq V_{j j}^{1 / 2} t_{n-p, 1-\alpha / 2}
$$

where $\mathrm{F}_{p, n-p, 1-\alpha}$ is the $100(1-\alpha) \%$ percentage point for the $\mathrm{F}$ distribution with $p$ and $n-p$ degrees of freedom, $t_{n-p, 1-\alpha / 2}$ is the $100(1-\alpha / 2) \%$ percentage point of the $t$ distribution with $n-p$ degrees of freedom, $V \in \Re^{(p+n m) \times(p+n m)}$ is the 
estimated linearized covariance matrix for the parameter estimators $\hat{\eta}$, and $V_{j j}^{1 / 2}$ is the square root of its $(j, j)$ th element.

The linearized covariance matrix for the estimators $\hat{\eta}$ is the $(p+n m) \times(p+n m)$ matrix

$$
\hat{V}=\hat{\sigma}^{2}\left[G^{\prime}(\hat{\eta})^{\mathrm{T}} \Omega G^{\prime}(\hat{\eta})\right]^{-1},
$$

where $G^{\prime}(\hat{\eta}) \in \Re^{(n+n m) \times(p+n m)}$ is the Jacobian matrix with $(i, j)$ th element equal to $\partial g_{i}(\eta) / \partial \eta_{j}$ evaluated at $\hat{\eta}$. (We assume that $G^{\prime}(\hat{\eta})$ is of full rank, so that $\left[G^{\prime}(\hat{\eta})^{\mathrm{T}} \Omega G^{\prime}(\hat{\eta})\right]$ is nonsingular.) In the next section, we show how the covariance matrix defined by (3.5) can be computed in a numerically stable way.

For nonlinear ordinary least squares, the linearization method is asymptotically correct as $n \rightarrow \infty$. (See, e.g., [Jennrich, 1969].) For the orthogonal distance regression problem, this method has been shown to be asymptotically correct as $\sigma^{a} \rightarrow 0$ [Fuller, 1987]. The difference between the conditions of asymptotic correctness can be explained by the fact that, as the number of observations increases in the orthogonal distance regression problem, one does not obtain additional information for $\delta_{i}$. Thus, for orthogonal distance regression problems, one would expect the portion of the covariance matrix concerned with $\beta$ to yield linearized regions and intervals as accurate as those computed for nonlinear ordinary least squares problems, while one would expect the regions and intervals for $\delta_{i}^{a}$ to be less accurate.

Note also that $\hat{V}$ is dependent upon the weight matrix $\Omega$, which must be assumed to be correct, and cannot be confirmed from the orthogonal distance regression results. Errors in the $w_{i}$ and $d_{i}$ that form $\Omega$ will have an adverse affect on the accuracy of $\hat{V}$ and its component parts. In $\S 5$, we present the results of a Monte Carlo experiment examining the accuracy of the linearized confidence intervals for four measurement error models. The results indicate that the confidence regions and intervals for $\delta_{i}^{a}$ are not as accurate as those for $\beta^{a}$. These results also show that errors in $\Omega$ can have an adverse affect on both confidence regions and intervals.

\section{Computing the Covariance Matrix $\hat{V}$}

The most straightforward computation of a quantity is often not the most numerically stable. Although $\hat{V}$ is defined as

$$
\hat{V}=\hat{\sigma}^{2}\left[G^{\prime}(\hat{\eta})^{\mathrm{T}} \Omega G^{\prime}(\hat{\eta})\right]^{-1},
$$


we would not compute it by first calculating $G^{\prime}(\hat{\eta})^{\mathrm{T}} \Omega G^{\prime}(\hat{\eta})$ and then inverting the resulting $(p+n m) \times(p+n m)$ matrix because such a procedure would introduce unnecessary numerical errors that could severely jeopardize the accuracy of $\hat{V}$. In this section, we present a numerically stable and efficient method for constructing $\hat{V}$.

For any $A \in \Re^{n \times p}$ with linearly independent columns, it is generally recommended that the matrix $\left[A^{\mathrm{T}} A\right]^{-1}$ be computed by first constructing the $Q R$ factorization of $A$, i.e., $A=Q R$, where $Q \in \Re^{n \times n}$ has orthonormal columns, and $R \in \Re^{n \times p}$ is upper triangular with positive diagonal elements. If we let $\tilde{R} \in \Re^{p \times p}$ be the upper $p \times p$ portion of $R$, then $A^{\mathrm{T}} A=R^{\mathrm{T}} R=\tilde{R}^{\mathrm{T}} \tilde{R}$ and $\left[A^{\mathrm{T}} A\right]^{-1}=\tilde{R}^{-1}\left(\tilde{R}^{-1}\right)^{\mathrm{T}}$. Since $\tilde{R}$ is triangular, its inverse can be accurately computed, thus allowing an accurate computation of $\left[A^{\mathrm{T}} A\right]^{-1}$. (See, e.g., [Dongarra et al., 1979].)

The computation of $\hat{V}$ can be further improved, however, since analysis of $G^{\prime}(\eta)$ shows that it has the special structure

$$
\left[\begin{array}{ll}
J & U \\
0 & I
\end{array}\right]
$$

where $J=F^{\prime}(\hat{\beta})$, and $U \in \Re^{n \times n m}$ is the "staircase" matrix

$$
\left[\begin{array}{cccc}
u_{1,1} \ldots u_{1, m} & & & \\
& u_{2, m+1} \ldots u_{2,2 m} & & \\
& \vdots & & \\
& & u_{n-1,1+m(n-2)} \ldots u_{n-1, m(n-1)} & \\
& & & u_{n, 1+m(n-1)} \ldots u_{n, n m}
\end{array}\right]
$$

with

$$
\begin{aligned}
u_{i, j} & =\partial g_{i}(\eta) / \partial \eta_{p+j} \\
& = \begin{cases}\partial f\left(x_{i}+\delta_{i} ; \beta\right) / \partial \delta_{i, j-(i-1) m} & \text { if } 1+(i-1) m \leq j \leq i m \\
0 & \text { otherwise }\end{cases}
\end{aligned}
$$

for $i=1, \ldots, n$ and $j=1, \ldots, n m$. Thus,

$$
\begin{aligned}
\hat{V} & =\hat{\sigma}^{2}\left[G^{\prime}(\hat{\eta})^{\mathrm{T}} \Omega G^{\prime}(\hat{\eta})\right]^{-1} \\
& =\hat{\sigma}^{2}\left[\left[\begin{array}{cc}
J^{\mathrm{T}} & 0 \\
U^{\mathrm{T}} & I
\end{array}\right]\left[\begin{array}{cc}
W & 0 \\
0 & D
\end{array}\right]\left[\begin{array}{cc}
J & U \\
0 & I
\end{array}\right]\right]^{-1} \\
& =\hat{\sigma}^{2}\left[\begin{array}{cc}
J^{\mathrm{T}} W J & J^{\mathrm{T}} W U \\
U^{\mathrm{T}} W J & U^{\mathrm{T}} W U+D
\end{array}\right]^{-1} .
\end{aligned}
$$


We can partition $\hat{V}$ as

$$
\hat{V}=\left[\begin{array}{cc}
\hat{V}_{\beta} & \hat{V}_{\beta \delta} \\
\hat{V}_{\delta \beta} & \hat{V}_{\delta}
\end{array}\right]
$$

where $\hat{V}_{\beta} \in \Re^{p \times p}$ is the covariance matrix of the estimated $\hat{\beta}_{\mathrm{s}}, \hat{V}_{\delta} \in \Re^{n m \times n m}$ is the covariance matrix of the estimated $\hat{\delta}$ s, and $\hat{V}_{\beta \delta}=\hat{V}_{\delta \beta}^{\mathrm{T}} \in \Re^{p \times n m}$ gives covariances between the $\beta \mathrm{s}$ and the $\delta \mathrm{s}$. The component parts of $\hat{V}$ are thus

$$
\begin{aligned}
& \hat{V}_{\beta}=\hat{\sigma}^{2}\left[J^{\mathrm{T}}\left(W-W U\left[U^{\mathrm{T}} W U+D\right]^{-1} U^{\mathrm{T}} W\right) J\right]^{-1} \\
& \hat{V}_{\beta \delta}=-\hat{\sigma}^{2} \hat{V}_{\beta}\left(J^{\mathrm{T}} W U\right)\left[U^{\mathrm{T}} W U+D\right]^{-1} \\
& \hat{V}_{\delta \beta}=\hat{V}_{\beta \delta}^{\mathrm{T}} \\
& \hat{V}_{\delta}=-\hat{\sigma}^{2}\left[U^{\mathrm{T}} W U+D\right]^{-1}\left(I+\left(U^{\mathrm{T}} W J\right) \hat{V}_{\beta}\left(J^{\mathrm{T}} W U\right)\left[U^{\mathrm{T}} W U+D\right]^{-1}\right) .
\end{aligned}
$$

The structural properties of the matrices appearing in (4.6) can be exploited to compute $\hat{V}$ accurately and efficiently. In Boggs et al. [1987], they define

$$
P^{-1} \equiv\left[U^{\mathrm{T}} W U+D\right]^{-1}
$$

and

$$
\omega_{i} \equiv \sum_{j=1}^{n m} \frac{u_{i, j}^{2}}{D_{j, j}} \quad i=1, \ldots, n
$$

They then show that

$$
P^{-1}=D^{-1}-D^{-1} U^{\mathrm{T}} W^{1 / 2} M W^{1 / 2} U D^{-1}
$$

where $M \in \Re^{n \times n}$ is the diagonal matrix defined by

$$
M \equiv \operatorname{diag}\left\{\left[\frac{1}{1+\omega_{i}}\right], i=1, \ldots, n\right\} .
$$

Because $D$ and $W$ are diagonal, $P^{-1}$ can be easily computed. Boggs et al. [1987] also show that

$$
W-W U\left[U^{\mathrm{T}} W U+D\right]^{-1} U^{\mathrm{T}} W=M .
$$

Thus, we can define the linearized covariance matrix as

$$
\begin{aligned}
\hat{V}_{\beta} & =\hat{\sigma}^{2}\left[J^{\mathrm{T}}\left(W-W U\left[U^{\mathrm{T}} W U+D\right]^{-1} U^{\mathrm{T}} W\right) J\right]^{-1} \\
& =\hat{\sigma}^{2}\left[\left(M^{1 / 2} J\right)^{\mathrm{T}}\left(M^{1 / 2} J\right)\right]^{-1}
\end{aligned}
$$


which can be stably computed using the $Q R$ factorization techniques described at the beginning of this section. This result and (4.7) allow the efficient formation of $\hat{V}_{\beta \delta}, \hat{V}_{\delta \beta}$ and $\hat{V}_{\delta}$.

The covariance matrix $\hat{V}_{\beta}$ of the estimators $\hat{\beta}$ provided by ODRPACK is computed using the above technique; in our experience, users are seldom interested in $\hat{V}_{\delta}$ or $\hat{V}_{\beta \delta}$. If necessary, however, the full covariance matrix $\hat{V}$ for all of the estimators $\hat{\eta}$ either can be computed using the above equations, or can be "automatically" obtained from most ordinary least squares software (including ODRPACK) by solving the orthogonal distance regression problem as the ordinary least squares problem defined by (2.4).

\section{Computational Experience}

Given the ability to define and construct linearized confidence regions and intervals for the parameters of an orthogonal distance regression problem, it is reasonable to ask how good these regions and intervals are. In this section, we present the results of a Monte Carlo study that indicates that, at least in some cases, linearized confidence regions and intervals are quite good.

A Monte Carlo experiment allows us to examine the properties of confidence regions and intervals for a given problem. For such an experiment, we define the observed coverage, $\hat{\gamma}_{\alpha}$, of a constructed confidence interval or region as the percentage of the time that true value lies within the interval or region constructed for the parameter. The nominal coverage of such a region or interval is $100(1-\alpha) \%$. When the number of realizations of the data is large, then the observed coverage will reflect the actual (or true) coverage, $\gamma_{\alpha}^{a}$, of the given region or interval. The actual coverage may or may not be the same as the nominal coverage, however. By comparing $\hat{\gamma}_{\alpha}$ with $100(1-\alpha) \%$, we can thus assess the quality of an approximate confidence interval or region.

Donaldson and Schnabel [1987] examined linearized confidence intervals and regions for a number of nonlinear ordinary least squares models and data sets. They found that the linearization method is not always adequate. Their results showed that, while the linearized confidence intervals were generally good, the linearization method confidence regions frequently resulted in observed coverage that was far lower than nominal.

We would not expect an exhaustive study of orthogonal distance regression problems to produce results that were substantially different than those found by Donaldson and Schnabel [1987]. Thus we do not attempt such a large scale 
study. Here we present the results of a Monte Carlo study of only four data sets. These results demonstrate that the linearized confidence intervals and regions can be quite reasonable when $\Omega$ is known precisely. When $\Omega$ is not known precisely, however, the study shows that the observed coverage of the linearized regions and intervals can differ significantly from the nominal value.

Our first example is from Fuller [1987, example 3.2.2, p. 230-238]. The data $\left(x_{i}, y_{i}\right)$ are the percent saturation of nitrogen gas in a brine solution forced into the pores of sandstone, and the observed compressional wave velocity of ultrasonic signals propagated through the sandstone, respectively. These data are assumed to be modeled by

$$
f\left(x_{i}+\delta_{i} ; \beta\right)=\beta_{1}+\beta_{2}\left(e^{\beta_{3}\left(x_{i}+\delta_{i}\right)}-1\right)^{2} .
$$

Fuller analyzed the original data assuming a measurement error model with $D^{a}=$ I. For our Monte Carlo experiment, we assigned

$$
\begin{aligned}
\beta^{a} & =(1264.65,-54.02,-0.0879)^{\mathrm{T}} \\
X^{a} & =(0.0,0.0,5.0,7.0,7.5,10.0,16.0,26.0,30.0,34.0,34.5,100.0)^{\mathrm{T}}, \\
w_{i}^{a} & =1, i=1, \ldots, 12 \\
d_{i}^{a} & =1, i=1, \ldots, 12 \\
\sigma^{a} & =\sqrt{2.38} .
\end{aligned}
$$

The values selected for $\beta^{a}$ and $\sigma^{a}$ are those estimated by Fuller using the original data with $\Omega=I$. The values $x_{i}^{a}, i=1, \ldots, n$, are the observations from the original experiment. Fuller notes that it is reasonable to believe that the error variance for $x=0$ and $x=100$ is smaller than the error variances for the remaining observations. For our Monte Carlo experiment, we assume that $x_{1}, x_{2}$ and $x_{12}$ are observed without error, and thus fix $\delta_{1}^{a}=\delta_{2}^{a}=\delta_{12}^{a}=0$.

Our second example is from Ratkowsky [1983, example 6.11, p. 119-120]. The response variable purports to represent resistance of a thermistor and the predictor variable temperature. Ratkowsky, however, notes that since the resistance of a thermistor increases with temperature, the response variable probably represents conductance. The model used to describe the original data is

$$
f\left(x_{i} ; \beta\right)=-\beta_{1}+\frac{\beta_{2}}{x_{i}+\beta_{3}} .
$$

The analysis by Ratkowsky assumed that there was no error in the response variable; for our results, we assume a measurement error model with $d_{i}^{a}=1 / 10, i=$ 
$1, \ldots, n$. For this example, we assigned

$$
\begin{aligned}
\beta^{a} & =(5.0,6150.0,350.0)^{\mathrm{T}}, \\
X^{a} & =(45+5 i, i=1, \ldots, 16)^{\mathrm{T}}, \\
w_{i}^{a} & =1, i=1, \ldots, 16 \\
d_{i}^{a} & =1 / 10, i=1, \ldots, 16 \\
\sigma^{a} & =0.0002 .
\end{aligned}
$$

The values $x_{i}^{a}, i=1, \ldots, n$, are the observations from the original experiment. The other values are approximately those obtained by Ratkowsky in his analysis.

The third example is problem E, chapter 10 of Draper and Smith [1981, p. 518519]. This example models the relationship between pressure and temperature in saturated steam using

$$
f\left(x_{i} ; \beta\right)=\beta_{1} \cdot 10^{\beta_{2} x_{i} /\left(\beta_{3}+x_{i}\right)} .
$$

Draper and Smith assumed that there was no error in the temperature observations; for our results, we assume a measurement error model with $d_{i}^{a}=10, i=$ $1, \ldots, n$. We assigned

$$
\begin{aligned}
\beta^{a} & =(4.18,6.91,205.0)^{\mathrm{T}}, \\
X^{a} & =(0,10,20,30,40,50,60,70,80,85,90,95,100,105)^{\mathrm{T}}, \\
w_{i}^{a} & =1, i=1, \ldots, 14 \\
d_{i}^{a} & =10, i=1, \ldots, 14 \\
\sigma^{a} & =1.2 .
\end{aligned}
$$

The values $x_{i}^{a}, i=1, \ldots, n$, are the observations from the original experiment. The other values are approximately those obtained using the measurement error model for the original data with $d_{i}^{a}=10$.

The data for the fourth example were collected as part of a psychophysical experiment to evaluate the ability of human subjects to perceive a visual signal as a function of the intensity of the signal. The predictor variable, $x_{i}$, represents the signal intensity and the response variable, $y_{i}$, is the fraction of the total number of trials during which a particular subject correctly identified the presence of the signal. Each signal level was repeated 80 times. A sigmoidal curve belonging to the family

$$
f\left(x_{i} ; \beta\right)=\frac{\beta_{1}}{\left[1+e^{\beta_{2}-\beta_{3}\left(x_{i}+\delta_{i}\right)}\right]^{\beta_{4}}}
$$


is used to relate $y$ to $x$. We assigned

$$
\begin{aligned}
\beta^{a} & =(0.936,3.400,339.370,0.954)^{\mathrm{T}} \\
X^{a} & =(0.003,0.007,0.008,0.010,0.015,0.026,0.038,0.060,0.065)^{\mathrm{T}}, \\
w_{i}^{a} & =\left(y_{i}^{a} \cdot\left(1-y_{i}^{a}\right) / 80\right)^{-1 / 2}, i=1, \ldots, 9 \\
d_{i}^{a} & =\frac{30\left(y_{i}^{a} \cdot\left(1-y_{i}^{a}\right) / 80\right)^{1 / 2}}{x_{i}^{a}}, i=1, \ldots, 9 \\
\sigma^{a} & =1.0 .
\end{aligned}
$$

The values $x_{i}^{a}, i=1, \ldots, n$, are the observed values from the original experiment. The weights $w_{i}$ are calculated as the inverses of the standard deviations of the $y_{i}$. The standard deviations of the errors in the measurements of the various signal levels are known from experience to be proportional to the value of the signal itself with a proportionality constant of $1 / 30$. The $d_{i}^{a}$ are computed accordingly.

These 4 examples are plotted in figures 1 through 4 , respectively. The graphs display $f\left(x ; \beta^{a}\right)$ evaluated over the range of the values $x_{i}^{a}, i=1, \ldots, n$. The $n$ points $\left(x_{i}^{a}, y_{i}^{a}\right)$ are indicated by the "dots" on each curve. For each of these models, we assume that the true values $\left(x_{i}^{a}, y_{i}^{a}\right)$ are fixed; thus, we are considering functional models in the terminology of Kendall and Stuart [1979].

We construct 500 sets of "observed" data $\left(x_{i}, y_{i}\right)$ for each model using

$$
\begin{aligned}
& x_{i}=\quad x_{i}^{a}-\delta_{i}^{a} \quad i=1, \ldots, n \\
& y_{i}=f\left(x_{i}^{a} ; \beta^{a}\right)-\epsilon_{i}^{a} \quad i=1, \ldots, n \text {. }
\end{aligned}
$$

The errors $\left(\epsilon_{1}^{a}, \ldots, \epsilon_{n}^{a}, \delta_{1}^{a}, \ldots, \delta_{n}^{a}\right) \sim \mathrm{N}\left(0,\left(\sigma^{a}\right)^{2}\left[\Omega^{a}\right]^{-1}\right)$, are generated using the Marsaglia and Tsang [1984] pseudonormal random number algorithm as implemented by James Blue and David Kahaner of the National Institute of Standards and Technology. We construct $\Omega^{a}$ using (2.3) and

$$
\begin{aligned}
W^{a} & =\operatorname{diag}\left\{\left(w_{i}^{a}\right)^{2}, i=1, \ldots, n\right\} \\
D^{a} & =\operatorname{diag}\left\{\left(w_{i}^{a} d_{i}^{a}\right)^{2}, i=1, \ldots, n\right\} .
\end{aligned}
$$

For each of the 500 realizations of the data, we solve for $\hat{\eta}=$ $\left(\hat{\beta}_{1}, \ldots, \hat{\beta}_{p}, \hat{\delta}_{1}, \ldots, \hat{\delta}_{n}\right)^{\mathrm{T}}$ using the orthogonal distance regression software library ODRPACK [Boggs et al., 1989] and the ordinary least squares representation of the problem. (Recall that ODRPACK only computes the covariance matrix for the estimators $\hat{\beta}$ when solving an orthogonal distance regression problem. Using 
the ordinary least squares representation of the problem allows us to easily obtain the covariance matrix for the all of the estimators $\hat{\eta}$.) The computations are performed in double precision Fortran on a Sun Workstation.

Initially, $\eta$ is set to $\eta^{a}=\left(\beta_{1}^{a}, \ldots, \beta_{p}^{a}, \delta_{1}^{a}, \ldots, \delta_{n}^{a}\right)^{\mathrm{T}}$. This is reasonable, since we are interested in assessing the reliability of the confidence intervals and not in ODRPACK's ability to obtain a solution quickly. The results presented would not change in any significant way if we were to choose starting values within some small enough region about the true values: given a starting value "close enough" to a local optimum, that local optimum will be located by ODRPACK to whatever accuracy is specified in the calling sequence. Clearly, not every starting value will necessarily produce the same local solution. This issue of non-uniqueness is addressed in Boggs et al. [1987]. Here, our use of the true parameter value as the starting value reduces the probability that alternate local optima will be found by the regression procedure, and therefore that the confidence interval and region results will be confounded by the existence such alternate optima. We recognize, however, that in practice one may need to examine the region about the solution for other local minimum.

Default values are used for all ODRPACK arguments, except for the maximum number of iterations, which is set to 100. The Jacobian matrices are computed using finite differences. We expect that use of analytic derivatives would produce a slight improvement in the results reported here.

The covariance matrix is dependent upon $\Omega$. Clearly, however, the $W$ and $D$ that make up $\Omega$ are not always known. Of particular interest for orthogonal distance regression problems is the case where the values of $d_{i}, i=1, \ldots, n$, used to determine $D$ are only approximate. In addition to reporting the observed coverage for $\Omega$ constructed using $d_{i}^{a}$ and $w_{i}^{a}$, we therefore also report the observed coverage when the 500 replications are solved using $\Omega$ constructed with $d_{i}=d_{i}^{a} / 10$, $d_{i}=d_{i}^{a} / 2, d_{i}=2 d_{i}^{a}, d_{i}=10 d_{i}^{a}$, and $d_{i}=\infty d_{i}^{a}$, the latter indicating an ordinary least squares solution in which all values of $\delta_{i}$ are forced to zero.

The observed coverages for these problems are shown in Tables 1,2,3, and 4. The confidence region coverage when $d_{i}$ is correct is surprisingly good when compared with that observed by Donaldson and Schnabel [1987]. We conjecture that this is due to our choice of examples, and is not a property of orthogonal distance regression in general. In their study, Donaldson and Schnabel frequently found that the observed coverage for linearized confidence regions was less than $80 \%$ of the expected nominal coverages, a difference that many, if not most, users would find unacceptable. Our results show such a significant degradation in the 
coverage of the linearized confidence regions when $d_{i}$ is incorrect by even a factor of 2 .

These tables indicate that the confidence interval coverage when $d_{i}$ is correct is very good. In addition, the confidence intervals for $\beta^{a}$ are still quite good when $d_{i}$ is known only to within a factor of 2 , but when $d_{i}$ is under-estimated by a factor of 10 , we see a significant degradation for two of our four examples. There is also a significant degradation in the confidence interval coverage for $\delta_{i}^{a}$ when $d_{i}$ is not known precisely. As expected, our results show that the confidence intervals for $\beta^{a}$ are in general more reliable than those for $\delta_{i}^{a}$.

One surprising result is that, for confidence intervals for $\beta^{a}$, over-estimation of $d_{i}$ is preferable to under-estimation. For $\delta_{i}^{a}$, the opposite is true. We believe that this occurs because when $d_{i}$ is over-estimated, we overly restrict the size of $\delta_{i}$ and thus prevent $\hat{\delta}_{i}$ from being "close enough" to $\delta_{i}^{a}$ to allow the confidence intervals and regions to include $\delta_{i}^{a}$. When we under-estimate $d_{i}$, on the other hand, we artificially reduce the size of the residual variance, $\hat{\sigma}^{2}$, and thus the size of the covariance matrix and the resulting confidence intervals for $\beta^{a}$.

We conclude from this small study that for at least some orthogonal distance regression problems, confidence regions and intervals constructed using the covariance matrix do have some validity, especially when $d_{i}$ is known at least to within a factor of 2 . We recognize, however, that for other problems such intervals and regions may be very inaccurate. We also recognize that there is nothing better that can be easily reported in their place. Thus, the linearization method will continue to be the most frequently implemented method in production least squares software. We therefore advocate the use of linearized confidence intervals and regions for measurement error problems in the same spirit, and with the same caveats, that they are used for nonlinear ordinary least squares problems.

Finally, this study illustrates how the observed coverage of the linearized confidence intervals and regions for the parameters of any model can be easily computed using a Monte Carlo study and an efficient orthogonal distance regression package such as ODRAPCK.

Acknowledgement. The authors thank H. Iyer, Colorado State University, for many useful discussions and comments concerning this work, and for providing the fourth example for our Monte Carlo study. 


\section{References}

[1] Bard, Y. (1974), Nonlinear Parameter Estimation, New York: Academic Press.

[2] Bates, D. M., and Watts, D. G. (1980), "Relative Curvature Measures of Nonlinearity," Journal of the Royal Statistical Society, Ser. B, 42, 1-25

[3] Boggs, P. T., Byrd, R. H., and Schnabel, R. B. (1987), "A Stable and Efficient Algorithm for Nonlinear Orthogonal Distance Regression," SIAM Journal of Scientific and Statatistical Computing, 8(6), 1052-1078.

[4] Boggs, P. T., Donaldson, J. R., Byrd, R. H. and Schnabel, R. B. (1989), "ODRPACK - Software for Weighted Orthogonal Distance Regression," ACM Transactions on Mathematical Software, 15(4), 348-364.

[5] Boggs, P. T., Donaldson, J. R., Schnabel, R. B., and Spiegelman, C. H. (1988), "A Computational Examination of Orthogonal Distance Regression," Journal of Econometrics, 38(1/2), 169-201.

[6] Donaldson, J. R., and Schnabel, R. B. (1987), "Computational Experience with Confidence Regions and Confidence Intervals for Nonlinear Least Squares," Technometrics, 29(1), 67-82.

[7] Dongarra, J. J., Moler, C. B., Bunch, J. R., and Stewart, G. W. (1979), LINPACK Users' Guide, Philadelphia: SIAM.

[8] Draper, N. R., and Smith, H. (1981), Applied Regression Analysis, Second Edition, New York: John Wiley and Sons.

[9] Efron, B. (1985), The Jackknife, the Bootstrap and Other Resampling Plans, Philadelphia: SIAM.

[10] Fuller, W. A. (1987), Measurement Error Models, New York: John Wiley and Sons.

[11] Jennrich, R. I. (1969), "Asymptotic Properties of Non-linear Least Squares Estimators," Annals of Mathematical Statistics, 40, 633-643.

[12] Kendall, M. G., and Stuart, A. (1979), The Advanced Theory of Statistics, Vol. 2: Inference and Relationship (4th ed.), London: Charles Griffin. 
[13] Ratkowsky, D. A. (1983), Nonlinear Regression Modeling, New York: Marcel Dekker. 
Table 1: Fuller Example 3.2.2

\begin{tabular}{l|cccccc} 
& \multicolumn{6}{|c}{ Observed Coverage } \\
& $d_{i}=\frac{1}{10} d_{i}^{a}$ & $d_{i}=\frac{1}{2} d_{i}^{a}$ & $d_{i}=d_{i}^{a}$ & $d_{i}=2 d_{i}^{a}$ & $d_{i}=10 d_{i}^{a}$ & $d_{i}=\infty d_{i}^{a}$ \\
& & & & & & $($ OLS) \\
\hline c.r. for $\eta^{a}$ & 16.8 & 78.4 & 93.6 & 86.6 & 0.0 & - \\
c.r. for $\beta^{a}$ & 42.8 & 85.0 & 93.8 & 96.6 & 95.4 & 95.2 \\
c.r. for $\delta^{a}$ & 18.4 & 82.6 & 93.4 & 81.2 & 0.0 & - \\
\hline c.i. for $\beta_{1}^{a}$ & 50.0 & 84.4 & 93.8 & 97.2 & 97.4 & 97.4 \\
c.i. for $\beta_{2}^{a}$ & 51.4 & 85.8 & 94.6 & 96.0 & 96.8 & 96.6 \\
c.i. for $\beta_{3}^{a}$ & 89.4 & 92.2 & 93.8 & 94.4 & 95.2 & 95.6 \\
\hline c.i. for $\delta_{3}^{a}$ & 68.8 & 89.4 & 96.0 & 92.4 & 25.4 & - \\
c.i. for $\delta_{4}^{a}$ & 77.8 & 89.6 & 93.6 & 92.4 & 33.0 & - \\
c.i. for $\delta_{5}^{a}$ & 80.0 & 89.8 & 94.4 & 91.8 & 27.6 & - \\
c.i. for $\delta_{6}^{a}$ & 85.0 & 91.2 & 97.0 & 93.0 & 31.0 & - \\
c.i. for $\delta_{7}^{a}$ & 88.2 & 92.6 & 94.4 & 87.4 & 27.8 & - \\
c.i. for $\delta_{8}^{a}$ & 93.0 & 93.8 & 92.2 & 83.6 & 25.8 & - \\
c.i. for $\delta_{9}^{a}$ & 89.0 & 96.2 & 95.8 & 85.8 & 28.6 & - \\
c.i. for $\delta_{10}^{a}$ & 90.0 & 94.6 & 93.4 & 82.8 & 31.2 & - \\
c.i. for $\delta_{11}^{a}$ & 89.0 & 95.4 & 93.4 & 85.6 & 30.6 & -
\end{tabular}


Table 2: Ratkowsky Example 6.11

\begin{tabular}{l|cccccc} 
& \multicolumn{6}{|c}{ Observed Coverage } \\
& \multicolumn{5}{|c}{ for Nominal 95\% Confidence Regions and Intervals } \\
& $d_{i}=\frac{1}{10} d_{i}^{a}$ & $d_{i}=\frac{1}{2} d_{i}^{a}$ & $d_{i}=d_{i}^{a}$ & $d_{i}=2 d_{i}^{a}$ & $d_{i}=10 d_{i}^{a}$ & $d_{i}=\infty d_{i}^{a}$ \\
& & & & & & $($ OLS) \\
\hline c.r. for $\eta^{a}$ & 0.0 & 99.6 & 96.2 & 28.0 & 0.0 & - \\
c.r. for $\beta^{a}$ & 94.6 & 93.6 & 93.4 & 93.4 & 93.4 & 93.4 \\
c.r. for $\delta^{a}$ & 0.0 & 100.0 & 96.0 & 21.4 & 0.0 & - \\
\hline c.i. for $\beta_{1}^{a}$ & 95.4 & 95.2 & 95.2 & 95.0 & 95.0 & 95.0 \\
c.i. for $\beta_{2}^{a}$ & 95.4 & 95.2 & 95.2 & 95.0 & 95.0 & 95.0 \\
c.i. for $\beta_{3}^{a}$ & 95.4 & 95.2 & 95.2 & 95.0 & 95.0 & 95.0 \\
\hline c.i. for $\delta_{1}^{a}$ & 97.8 & 99.6 & 95.0 & 69.4 & 14.4 & - \\
c.i. for $\delta_{2}^{a}$ & 87.8 & 99.0 & 96.2 & 74.2 & 18.2 & - \\
c.i. for $\delta_{3}^{a}$ & 82.8 & 99.8 & 94.6 & 69.2 & 17.6 & - \\
c.i. for $\delta_{4}^{a}$ & 73.0 & 99.0 & 95.2 & 70.0 & 15.4 & - \\
c.i. for $\delta_{5}^{a}$ & 73.8 & 99.4 & 93.2 & 70.8 & 17.4 & - \\
c.i. for $\delta_{6}^{a}$ & 71.4 & 99.2 & 94.4 & 74.4 & 16.4 & - \\
c.i. for $\delta_{7}^{a}$ & 75.0 & 99.2 & 95.4 & 75.0 & 15.4 & - \\
c.i. for $\delta_{8}^{a}$ & 73.8 & 99.6 & 95.2 & 73.8 & 17.6 & - \\
c.i. for $\delta_{9}^{a}$ & 72.2 & 99.2 & 95.2 & 72.6 & 18.2 & - \\
c.i. for $\delta_{10}^{a}$ & 75.4 & 99.6 & 96.4 & 70.6 & 19.0 & - \\
c.i. for $\delta_{11}^{a}$ & 69.4 & 98.2 & 94.2 & 74.2 & 18.8 & - \\
c.i. for $\delta_{12}^{a}$ & 69.8 & 99.0 & 94.4 & 72.6 & 20.6 & - \\
c.i. for $\delta_{13}^{a}$ & 69.6 & 99.0 & 95.8 & 68.2 & 16.8 & - \\
c.i. for $\delta_{14}^{a}$ & 76.2 & 99.0 & 94.0 & 71.0 & 14.6 & - \\
c.i. for $\delta_{15}^{a}$ & 84.0 & 99.8 & 95.4 & 73.0 & 14.4 & - \\
c.i. for $\delta_{16}^{a}$ & 95.6 & 99.6 & 95.0 & 70.6 & 17.0 & - \\
& & & & & & \\
\end{tabular}


Table 3: Draper and Smith Problem 10.E

\begin{tabular}{|c|c|c|c|c|c|c|}
\hline & \multicolumn{6}{|c|}{$\begin{array}{l}\text { Observed Coverage } \\
\% \text { Confidence Regions and Intervals }\end{array}$} \\
\hline c.r. for $\eta^{a}$ & 13.2 & 85.0 & 92.4 & 72.2 & 0.0 & - \\
\hline c.r. for $\beta^{a}$ & 80.4 & 92.6 & 90.8 & 87.4 & 78.0 & 76.8 \\
\hline c.r. for $\delta^{a}$ & 28.6 & 94.0 & 94.8 & 68.0 & 0.0 & - \\
\hline c.i. for $\beta_{1}^{a}$ & 71.8 & 89.8 & 94.8 & 96.0 & 96.6 & 96.4 \\
\hline c.i. for $\beta_{2}^{a}$ & 86.6 & 93.2 & 94.0 & 91.8 & 89.4 & 89.6 \\
\hline c.i. for $\beta_{3}^{a}$ & 82.2 & 92.6 & 95.0 & 93.6 & 93.0 & 92.8 \\
\hline c.i. for $\delta_{1}^{a}$ & 100.0 & 98.8 & 94.4 & 82.2 & 29.2 & - \\
\hline c.i. for $\delta_{2}^{a}$ & 99.8 & 99.2 & 93.2 & 82.2 & 30.0 & - \\
\hline c.i. for $\delta_{3}^{a}$ & 92.6 & 99.2 & 94.6 & 78.8 & 25.0 & - \\
\hline c.i. for $\delta_{4}^{a}$ & 77.2 & 98.2 & 94.4 & 79.4 & 25.4 & - \\
\hline c.i. for $\delta_{5}^{a}$ & 82.6 & 97.0 & 94.4 & 79.8 & 26.2 & - \\
\hline c.i. for $\delta_{6}^{a}$ & 93.8 & 97.4 & 95.4 & 81.0 & 25.8 & - \\
\hline c.i. for $\delta_{7}^{a}$ & 96.8 & 96.0 & 96.0 & 83.4 & 25.8 & - \\
\hline c.i. for $\delta_{8}^{a}$ & 96.2 & 97.2 & 95.6 & 83.2 & 27.6 & - \\
\hline c.i. for $\delta_{9}^{a}$ & 98.2 & 97.0 & 96.0 & 85.6 & 23.8 & - \\
\hline c.i. for $\delta_{10}^{a}$ & 98.2 & 96.4 & 95.8 & 88.2 & 27.8 & - \\
\hline c.i. for $\delta_{11}^{a}$ & 99.4 & 96.0 & 95.4 & 90.2 & 28.0 & - \\
\hline c.i. for $\delta_{12}^{a}$ & 99.6 & 97.4 & 94.0 & 87.0 & 26.8 & - \\
\hline c.i. for $\delta_{13}^{a}$ & 100.0 & 98.6 & 95.2 & 85.6 & 25.2 & - \\
\hline c.i. for $\delta_{14}^{a}$ & 99.8 & 98.4 & 94.8 & 86.6 & 26.8 & - \\
\hline
\end{tabular}


Table 4: Psychophysical Example

\begin{tabular}{l|cccccc} 
& \multicolumn{6}{|c}{ Observed Coverage } \\
& $d_{i}=\frac{1}{10} d_{i}^{a}$ & $d_{i}=\frac{1}{2} d_{i}^{a}$ & $d_{i}=d_{i}^{a}$ & $d_{i}=2 d_{i}^{a}$ & $d_{i}=10 d_{i}^{a}$ & $d_{i}=\infty d_{i}^{a}$ \\
& & & & & & $(\mathrm{OLS})$ \\
\hline c.r. for $\eta^{a}$ & 46.6 & 59.4 & 57.4 & 37.6 & 0.0 & - \\
c.r. for $\beta^{a}$ & 53.8 & 52.0 & 48.2 & 47.6 & 46.4 & 46.4 \\
c.r. for $\delta^{a}$ & 98.0 & 100.0 & 95.8 & 59.4 & 0.0 & - \\
\hline c.i. for $\beta_{1}^{a}$ & 86.0 & 94.8 & 95.0 & 95.2 & 95.4 & 95.4 \\
c.i. for $\beta_{2}^{a}$ & 100.0 & 99.8 & 99.8 & 99.8 & 99.8 & 99.8 \\
c.i. for $\beta_{3}^{a}$ & 93.4 & 96.6 & 95.8 & 95.4 & 95.4 & 95.4 \\
c.i. for $\beta_{4}^{a}$ & 99.8 & 93.0 & 90.2 & 89.4 & 89.0 & 89.0 \\
\hline c.i. for $\delta_{1}^{a}$ & 100.0 & 99.6 & 95.0 & 75.6 & 22.0 & - \\
c.i. for $\delta_{2}^{a}$ & 99.8 & 99.6 & 96.4 & 78.2 & 22.4 & - \\
c.i. for $\delta_{3}^{a}$ & 100.0 & 99.4 & 95.2 & 76.4 & 20.2 & - \\
c.i. for $\delta_{4}^{a}$ & 100.0 & 99.4 & 96.4 & 75.8 & 19.4 & - \\
c.i. for $\delta_{5}^{a}$ & 100.0 & 99.8 & 95.0 & 73.6 & 18.2 & - \\
c.i. for $\delta_{6}^{a}$ & 99.8 & 99.4 & 95.0 & 75.0 & 18.8 & - \\
c.i. for $\delta_{7}^{a}$ & 98.8 & 99.6 & 95.6 & 77.2 & 17.8 & - \\
c.i. for $\delta_{8}^{a}$ & 100.0 & 99.4 & 94.8 & 72.2 & 20.4 & - \\
c.i. for $\delta_{9}^{a}$ & 100.0 & 99.4 & 95.2 & 77.6 & 21.0 & -
\end{tabular}

